\title{
Factores asociados a disfunción sexual durante el embarazo: estudio multicéntrico en la ciudad de Medellín
}

\section{Factors associated with sexual dysfunction during pregnancy: multi-center study in the city of Medellín}

María Isabel Jaramillo-Saldarriaga ${ }^{1}$, Alejandro Colonia-Toro ${ }^{2}$, Gina Andrea Posada ${ }^{3}$ María del Pilar Uribe

\section{RESUMEN}

El embarazo y el parto es un período especial en la vida de la mujer, ya que tiene implicaciones físicas, hormonales y psicológicas, además de cambios sociales y culturales que pueden influir en su sexualidad. Múltiples factores se han asociado con un aumento en la frecuencia de disfunción sexual durante la gestación y el posparto, la cual puede alcanzar una incidencia hasta del 50\%

\begin{abstract}
Pregnancy and childbirth are a special period in a woman's life, since it has physical, hormonal and psychological implications, in addition to the social and cultural changes that can influence her sexuality. Multiple factors have been associated with an increase in the frequency of sexual dysfunction during pregnancy and postpartum, which can reach
\end{abstract}

\section{Historial del artículo:}

Fecha de recepción: 29/09/2020

Fecha de aprobación: 12/03/2021

1 Universidad CES, Ginecóloga y Obstetra. Medellín, Colombia.

2 Universidad CES, Ginecólogo y Obstetra, Hospital General de Medellín. Medellín, Colombia.

3 Universidad CES, Ginecóloga Infantojuvenil, Clínica Medellín. Medellín, Colombia.

4 Universidad CES, Ginecóloga y Obstetra, Clínica El Rosario. Medellín, Colombia.

Correspondencia: María Isabel Jaramillo-Saldarriaga. Universidad CES, Cl. 10a \#22 - 04, Medellín, Colombia. Correo electrónico: mariaisajs@hotmail.com

Como citar este artículo: Jaramillo-Saldarriaga MI, Colonia-Toro A, Posada GA, Uribe MP. Factores asociados a disfunción sexual durante el embarazo: estudio multicéntrico en la ciudad de Medellín. Revista de la Facultad de Ciencias de la Salud de la Universidad del Cauca. 2021;23(1):15-27. https://doi.org/10.47373/rfcs.2021.v23.1626 
en estas etapas. Objetivo: Evaluar la prevalencia de disfunción sexual durante la gestación y su relación con algunos factores de riesgo y describir el comportamiento sexual de las mujeres embarazadas y postparto. Métodos: Estudio de corte transversal de pacientes embarazadas y postparto inmediato de tres centros médicos de atención obstétrica de la ciudad de Medellín. Resultados: Por medio de una encuesta personal se evaluaron 259 mujeres en embarazo y posparto en 3 diferentes centros de atención obstétrica de la ciudad de Medellín. Se encontró una prevalencia de disfunción sexual femenina del 60,2\%. El tipo más frecuente de relaciones sexuales fue la vaginal (69\%). El 85\% de las madres y el 71,4\% de los padres consideraban que existía riesgo de un posible daño al bebe con las relaciones sexuales. $\mathrm{El}$ 59,1\% de las embarazadas recibieron información sobre sexualidad durante la gestación, siendo el 30\% por parte del médico y se asoció como factor protector para disfunción sexual, junto con la estabilidad de la pareja. Como factores de riesgo se identifió el régimen subsidiado del sistema de seguridad social y la idea de dańo al embarazo por parte de la madre. Conclusión: La disfunción sexual es una entidad con una mayor prevalencia y algunas consideraciones especiales durante el embarazo, comparado con la población general. Debido a esto, los profesionales de la salud deben explorar este aspecto en la atención prenatal y brindar una adecuada información sobre la seguridad de las relaciones sexuales durante el embarazo y los posibles cambios en el deseo, el alcance del orgasmo y la satisfacción sexual.

Palabras claves: Sexualidad, Embarazo, Comportamiento, Sexual, Disfunción. (DeCS)

\section{INTRODUCCIÓN}

La sexualidad es un aspecto universal para todos los seres humanos; sin embargo, el comportamiento sexual se ve afectado por el contexto cultural o social de cada individuo (1). El embarazo siendo un período especial en la vida de la mujer, por sus implicaciones físicas, hormonales y psicológicas, puede modificar también este comportamiento $(2,3)$. Durante todo el embarazo la función sexual femenina registra una disminución significativa, especialmente en el tercer trimestre y que persiste hasta los primeros 3 a 6 meses luego del parto $(4,5)$, en este último período el dolor genital persistente cumple un papel importante (6). an incidence of up to 50\%. Objective: To describe the estimate sexual disfunction prevalence, and to describe sexual behavior of pregnant and postpartum women, and to explore what factors influence the female sexual function index. Methods: Cross-sectional study of pregnant and immediate postpartum patients from three obstetric care medical centers in the city of Medellin. Results: Through a personal survey, 259 pregnant and postpartum women were evaluated in 3 different obstetric care centers in the city of Medellin. $59.1 \%$ of the pregnant women received information about sexuality during pregnancy, 30\% being from the doctor and it was associated as a protective factor for sexual dysfunction, together with the stability of the couple. As risk factors, the subsidized regime of the social security system and the idea of harm to pregnancy by the mother were identified. Conclusion: Sexual dysfunction is an entity with a higher prevalence and some special considerations during pregnancy, compared to the general population. Because of this, health professionals should explore this aspect in prenatal care and provide adequate information on the safety of sexual intercourse during pregnancy and the possible changes in desire, reaching orgasm, and sexual satisfaction.

Keywords: Sexuality, Pregnancy, Behavior, Sexual, Dysfunction. (MeSH)
Todo esto hace que sea importante indagar sobre la disfunción sexual femenina durante la gestación y el puerperio, y aunque se comienzan a reversar paulatinamente los cambios físicos provocados por el embarazo, aparecen temores generados por la paternidad, el dolor y recuerdo de sensaciones desagradables. La vía del parto es un factor determinante; cuando el parto es instrumentado, hay un expulsivo prolongado o se presentan desgarros de alto grado, existe una mayor tasa de disfunción sexual materna (7-9). Sin embargo, con orientación adecuada hasta el 90\% de las pacientes reactivan su vida sexual a los tres meses postparto $(10,11)$, aunque este lapso tiempo es variable y se ve afectado por los factores ya mencionados y el tiempo de lactancia (12). 
En la población no embarazada, se ha reportado pérdida del deseo sexual hasta en el 30-35\% de las mujeres entre los 18 y 70 ańos (13). Según algunos reportes y estudios, la disfunción sexual femenina durante el embarazo puede alcanzar una prevalencia mayor del $50 \%$, siendo la principal causa la disminución de la sensibilidad del clítoris $(94,2 \%)$, seguido por la falta de libido $(92,6 \%)$ y el trastorno orgásmico (81\%) (14). Las razones defendidas como responsables de la reducción de la sexualidad durante embarazo son tres principalmente: preocupaciones sobre un parto prematuro, dańo al feto y las infecciones vaginales (15).

Dado que no se identificaron datos locales acerca de esta patología en esta etapa de la vida, el objetivo de este estudio fue evaluar la prevalencia de disfunción sexual durante la gestación y su relación con algunos factores de riesgo, con el fin de profundizar en el conocimiento de la sexualidad y sus implicaciones durante el embarazo.

\section{MÉTODOS}

Se realizó un estudio de corte transversal con pacientes embarazadas y postparto inmediato de tres centros médicos de atención obstétrica de la ciudad de Medellín que consultaron para atención obstétrica (control prenatal, atención del parto y consulta de urgencias), postparto inmediato y control postparto; quienes aceptaron de manera libre y espontánea participar en el estudio.

Se obtuvo la información de 259 pacientes a través de un muestreo por conveniencia y tomando las pacientes que aceptaran participar en el estudio durante el periodo del 1 al 31 de enero de 2016 y se seleccionaron por aparición consecutiva por consulta o parto en cada institución.

Fueron criterios de inclusión: Mujeres con edad gestacional mayor o igual a 26 semanas, por fecha de última menstruación confiable o por ecografía del primer trimestre que consultan en el Hospital General de Medellín, en Clínica SOMA o Medicina Fetal, dado que a partir de esta edad gestacional son más marcados los cambios asociados a la sexualidad; además, debían tener al momento del ingreso al estudio una pareja que conviviera con ella o una relación estable donde se vieran de manera frecuente. Se excluyeron las pacientes embarazadas con diagnóstico de patologías que pudieran disminuir o evitar la actividad sexual (ruptura de membranas, placenta previa, amenaza de aborto o parto pretérmino).
Se utilizó el cuestionario Índice de Función Sexual Femenina (IFSF) (16), validadas para su uso en espańol (12), el cual cuenta con 19 preguntas, cada pregunta tiene 5 o 6 opciones, asignándoles un puntaje que va de 0 a 5 . El puntaje de cada dominio se multiplica por un factor y el resultado final es la suma aritmética de los dominios. A mayor resultado, mayor nivel de satisfacción.

Se aplió también un cuestionario elaborado por los investigadores para obtener las variables demográficas y la información relacionadas con su comportamiento sexual. Se ingresaron los datos en una base de datos previamente diseńada con las variables requeridas en el programa SPSS V.21. Se realizó un análisis estadístico descriptivo. Las variables cuantitativas se reportaron en forma de medias y medianas según su distribución con su desviación estándar o rango intercuartil, y en cuanto a las cualitativas en forma de frecuencias y proporciones. Se calcularon OR crudos para las variables cualitativas y la significación con la prueba Chi cuadrado, considerando estadísticamente significativa una $p$ menor de 0.05. En todos los datos se calcularon intervalos de confianza del $95 \%$.

Se garantizó en todo momento la confidencialidad de los datos y se obtuvo consentimiento informado de todas las pacientes. El estudio fue presentado y aprobado por el comité de ética de las tres instituciones participantes: Medicina fetal SAS, Clínica SOMA y Hospital General de Medellín.

\section{RESULTADOS}

Se obtuvo información de 259 mujeres en embarazo y postparto inmediato, indagando sobre la función sexual en el último trimestre del embarazo. El promedio de edad de las pacientes fue de 25 ańos y la mitad de las pacientes tenían 34 semanas o más. Con respecto a la seguridad social, la mitad de las pacientes tenían régimen contributivo. El 95\% de las pacientes estaban entre el estrato 1 y 3 según la clasificación socioeconómica del sistema de seguridad social de Colombia y el $41 \%$ tenían estudios inferiores a bachillerato completo. Más de la mitad de las pacientes convivían en unión libre. La patología más frecuente asociada al embarazo fue el trastorno hipertensivo el cual se presentó en el 14\% de las pacientes (Tabla 1).

El 59,1\% de las embarazadas recibieron algún tipo de información sobre sexualidad durante su gestación, el medio más frecuente fue el médico en el $30 \%$ de los casos, seguido de personal de salud no médico en el 13,1\% (Figura 1). 
Figura 1. Proporción de las diferentes fuentes de información sobre sexualidad en el embarazo.

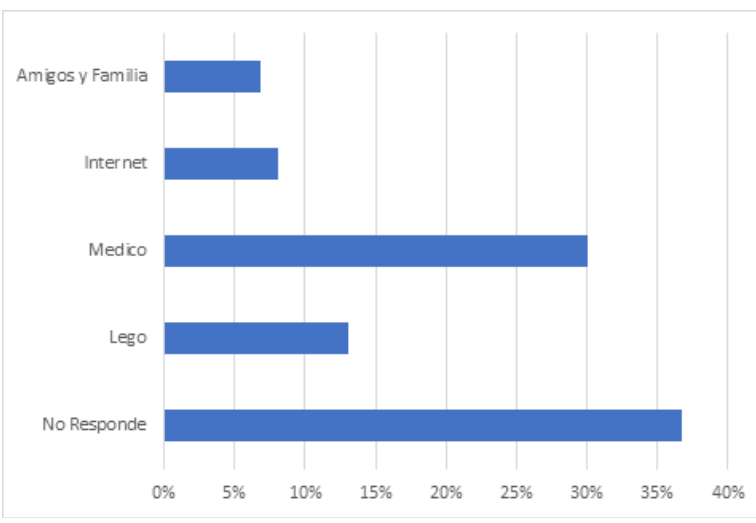

Con respecto a las conductas sexuales se encontró que el tipo de relaciones más frecuentes fue la vía vaginal en el $69 \%$ de las encuestadas y en segundo lugar la combinación de vaginal y oral (9,3\%). La mitad de las parejas tenían una frecuencia de encuentro sexual igual o mayor a $1 \mathrm{vez}$ a la semana y $89 \%$ de las pacientes refirió tener pareja sexual estable. Al evaluar sobre la creencia de un posible dańo al feto con las relaciones sexuales, la respuesta fue positiva en el $85 \%$ de las madres y el $71,4 \%$ refirió que existían temores por parte de su pareja.

Con respecto a los parámetros evaluados en el índice de disfunción sexual femenina en el embarazo se encontró:

Deseo Sexual: El 16\% refirieron nunca o casi nunca tener deseo sexual y el $12 \%$ siempre o casi siempre. Con respecto a la intensidad del deseo el $14 \%$ de las mujeres dijeron haber estado muy satisfechas con su intensidad.

Excitación: El 33\% manifestaron excitación la mayoría de las veces o siempre y el $29 \%$ nunca o pocas veces. El nivel de excitación fue alto o muy alto en el $21 \%$ de los casos.

Lubricación: El 52\% no tuvieron problemas con la lubricación, manifestando tenerla la mayoría de las veces o casi siempre, un $49,4 \%$ de las pacientes lograron mantener la lubricación en gran medida (49,4\%) siendo adecuada hasta el final de la relación en el 60\%. El 21\% manifestaron que la lubricación fue difícil o imposible.

Orgasmo: Un 39\% de las pacientes tuvieron orgasmos la mayoría o casi siempre en sus relaciones sexuales y el $26,4 \%$ nunca o pocas veces. El $43 \%$ de las mujeres gestantes manifestaron dificultades para lograrlo. El 49\% se sintieron satisfechas con sus orgasmos y el 17,4\% les fue indiferente.
Tabla 1. Características sociodemográficas de pacientes entrevistadas durante el embarazo y puerperio inmediato.

\begin{tabular}{ll}
\hline \multicolumn{1}{c}{$\begin{array}{c}\text { Variables } \\
\text { sociodemográficas }\end{array}$} & \multicolumn{1}{c}{$\begin{array}{c}\text { n (\%) media (DE) } \\
\text { mediana (RIQ) }\end{array}$} \\
\hline \multicolumn{2}{c}{ Social } \\
\hline \multicolumn{2}{c}{ Régimen de seguridad } \\
\hline Subsidiado \\
Contributivo \\
Otros $\quad 113(43,6 \%)$ \\
\hline \multicolumn{1}{c}{ Nivel educativo } & $138(53,3 \%)$ \\
\hline Secundaria incompleta 0 & $8(2,9 \%)$ \\
menos & $107(41,3 \%)$ \\
Secundaria completa & $90(34,7 \%)$ \\
\hline \multicolumn{1}{c}{ Estado civil } & \\
\hline Soltera & $55(21,2 \%)$ \\
Casada & $27(10,4 \%)$ \\
Unión Libre & $174(67,2 \%)$ \\
\hline \multicolumn{1}{c}{ Estrato social } \\
pretérmino paridad
\end{tabular}

\begin{tabular}{ll}
\hline $\begin{array}{c}\text { Recibió información sobre } \\
\text { sexualidad en embarazo }\end{array}$ \\
\hline $\mathrm{Si}$ & $153(59,1 \%)$ \\
$\mathrm{No}$ & $96(37,1 \%)$ \\
Edad materna & $25,3(\mathrm{DE} 6,5)$ \\
Edad gestacional & $34(\mathrm{RIQ} 8)$ \\
\hline
\end{tabular}

* DE: Desviación estándar

* RIQ: Rango intercuartil 
Satisfacción: El 53,3\% de las pacientes se sintieron satisfechas con la cercanía emocional de su pareja. Al preguntar por su satisfacción en general con su vida sexual el $31,7 \%$ dijeron estar muy satisfechas y el $8,9 \%$ muy insatisfecha (Figura 2).

Dolor: El 37\% presentaron algún grado de dispareunia durante la penetración, y el 12\% siempre o casi siempre. Sin embargo, el 66\% refirieron poco dolor posterior al coito. En general la intensidad del dolor durante la actividad sexual fue calificada como alta o muy alta en el 10,4\% y moderada en el $28 \%$ de ellas.

Índice de disfunción sexual femenina en el embarazo: La media en el índice fue de 24,2 . Se tomó como disfunción sexual una puntuación menor de 26 encontrando una prevalencia de $60,2 \%$ en este aspecto.

Se identificó al régimen subsidiado de salud y a la idea de un posible dańo fetal por la actividad sexual, como factores de riesgo para disfunción sexual. Por otro lado, se consideró como factor protector el recibir información sobre sexualidad en el embarazo y tener pareja estable. No hubo diferencia significativa con respecto a la edad de la madre, nivel educativo, edad gestacional al momento de la entrevista, ni el estrato socioeconómico. Las pacientes con disfunción sexual durante el embarazo tuvieron persistentes puntuaciones bajas en todos los ítems de evaluación y difirieron significativamente de las pacientes sin disfunción sexual en la gestación (Tabla 2).

Tabla 2. Factores asociados a disfunción sexual femenina durante la gestación según IDSF

\begin{tabular}{|c|c|c|c|c|}
\hline \multirow[t]{2}{*}{1} & \multicolumn{2}{|c|}{$\begin{array}{l}\text { Disfunción sexual (IDSF) } \\
\text { n (\%), Mediana (RIQ) }\end{array}$} & \multirow[t]{2}{*}{ OR (IC 95\%) } & \multirow[t]{2}{*}{ Valor $p$} \\
\hline & $<26$ & $\geq 26$ & & \\
\hline $\begin{array}{l}\text { Régimen subsidiado } \\
\mathrm{Si} \\
\text { No }\end{array}$ & $\begin{array}{l}80(70,8 \%) \\
73(52,1 \%)\end{array}$ & $\begin{array}{l}33(29.2 \%) \\
67(47,9 \%)\end{array}$ & $2,2(1,31-3,76)$ & 0,003 \\
\hline $\begin{array}{l}\text { Nivel educativo } \\
\text { Bachillerato incompleto o menos } \\
\text { Bachillerato completo }\end{array}$ & $\begin{array}{l}91(64,1 \%) \\
65(55,6 \%)\end{array}$ & $\begin{array}{l}51(35,9 \%) \\
52(44,4 \%)\end{array}$ & $1,42(0,87-2,35)$ & 0,16 \\
\hline $\begin{array}{l}\text { Número de hijos (incluye actual) } \\
1 \\
2 \text { ó mas }\end{array}$ & $\begin{array}{l}42(65,6 \%) \\
113(58,9 \%)\end{array}$ & $\begin{array}{l}22(34,4 \%) \\
79(41,1 \%)\end{array}$ & $1,33(0,74-2,41)$ & 0,34 \\
\hline $\begin{array}{l}\text { Información sobre sexualidad. } \\
\text { Si } \\
\text { No }\end{array}$ & $\begin{array}{l}83(54,2 \%) \\
67(69,8 \%)\end{array}$ & $\begin{array}{l}70(45,8 \%) \\
29(30,2 \%)\end{array}$ & $0,51(0,3-0,88)$ & 0,015 \\
\hline $\begin{array}{l}\text { Pareja estable } \\
\mathrm{Si} \\
\text { No }\end{array}$ & $\begin{array}{l}130(56,3 \%) \\
21(95,5 \%)\end{array}$ & $\begin{array}{l}101(43,7 \%) \\
1(4,5 \%)\end{array}$ & $0,06(0,008-0,46)$ & 0,001 \\
\hline $\begin{array}{l}\text { Idea de dańo materno por la madr } \\
\text { Si } \\
\text { No }\end{array}$ & $\begin{array}{l}26(83,9 \%) \\
124(56,1 \%)\end{array}$ & $\begin{array}{l}5(16,1 \%) \\
97(43,9 \%)\end{array}$ & $4,07(1,5-10,98)$ & 0,002 \\
\hline $\begin{array}{l}\text { Idea de dańo por parte del padre } \\
\text { Si } \\
\text { No }\end{array}$ & $\begin{array}{l}37(60,7 \%) \\
106(57,3 \%)\end{array}$ & $\begin{array}{l}24(39,3 \%) \\
79(42,7 \%)\end{array}$ & $1,15(0,64-2,07)$ & 0,65 \\
\hline Edad materna & $24(11)$ & $25(8)$ & & 0,36 \\
\hline Edad gestacional en la entrevista & $34(8)$ & $34(9)$ & & 0,95 \\
\hline
\end{tabular}


Figura 2. Proporción de la percepción individual de satisfacción sexual durante la gestación. (Independiente del ISFM)

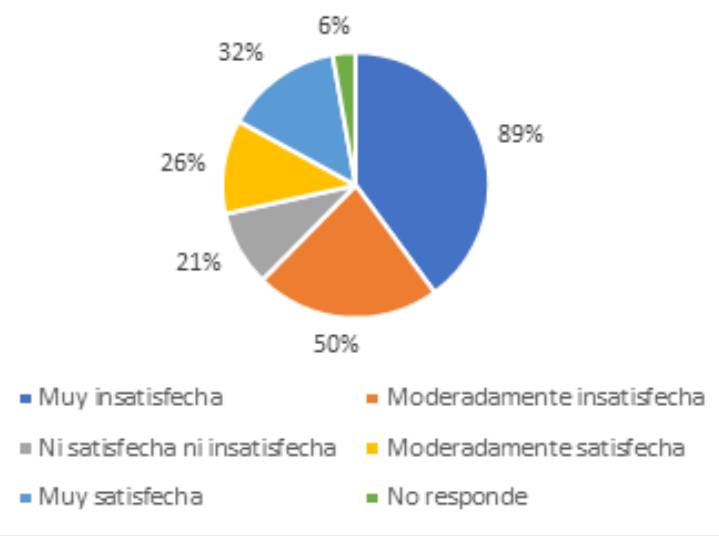

\section{DISCUSIÓN}

El embarazo puede alterar varios aspectos de la vida reproductiva de las mujeres, incluyendo la función sexual, tal como se pudo evidenciar en los resultados de este trabajo. Existen múltiples factores que pueden conducir a la disfunción sexual durante el embarazo, entre los cuales se encuentran los cambios físicos y hormonales que pueden causar una pérdida de lo que se percibe como atractivo y una disminución de la autoestima debido a cambios en la imagen corporal y otros factores psicológicos $(5,17)$. Se ha reportado que entre un 25$50 \%$ de las pacientes gestantes se sienten menos atractivas durante el embarazo (18).

De la misma manera, se ha visto que el contexto sociodemográfico de las pacientes puede afectar la sexualidad durante el embarazo (4). Nuestra población estuvo constituida principalmente por mujeres jóvenes de bajo nivel socioeconómico, esto podría influir en los resultados del estudio, posiblemente por la dificultad en la comprensión de las preguntas, la información previa respecto al tema y el acceso a los servicios de salud.

Se encontró una diferencia en los puntajes de disfunción sexual entre las pacientes que tenían una pareja estable y las que no. Según las estadísticas reportadas por el DANE (Departamento Administrativo Nacional de Estadísticas de Colombia) en el 2018, el 16,5\% de las pacientes gestantes en Colombia están solteras o separadas, lo que podría contribuir a la aparición de problemas y dificultades en el comportamiento sexual y algún grado de disfunción en nuestras pacientes embarazadas.

Casi la mitad de las pacientes recibieron información sobre sexualidad durante la gestación, de las cuales sólo el 30\% fue por parte del personal médico (Figura 1). Las pacientes informadas presentan menos disfunción sexual, lo cual podría sugerir la necesidad de incluir programas de educación sobre sexualidad en la consulta de control prenatal. Es importante que las pacientes tengan claro que las relaciones sexuales durante un embarazo de bajo riesgo son seguras y, además, que los cambios físicos y hormonales pueden generar fluctuaciones en el deseo y la satisfacción sexual $(3,19)$.

Existen varios reportes en la literatura que manifiestan la preocupación por parte de los padres sobre un posible dańo fetal con las relaciones sexuales. Un estudio realizado en Canadá encontró que hasta el $49 \%$ de las mujeres tienen la idea que las relaciones sexuales puede dańar el embarazo; siendo más frecuente la preocupación por presentar parto pretérmino en el tercer trimestre (15). Otro estudio realizado en Turquía en 2016, reportó que a un 38\% de las pacientes les preocupaba tener relaciones sexuales durante el embarazo, por miedo al dolor o a pérdida de la gestación (17). Llama la atención que en ambos estudios el porcentaje es menor que el encontrado en este trabajo; sin embargo, no tuvieron en cuenta la posible influencia de esta creencia en la pareja, en nuestro caso, este porcentaje de preocupación fue del $71,4 \%$.

Para evaluar adecuadamente la disfunción sexual utilizamos el Índice de Función Sexual Femenina (IFSF), el cual es un instrumento específico y multidimensional, que permite comparación de forma objetiva con los resultados de otros estudios. El valor utilizado del IFSF previamente, ha variado entre 26 a 30 puntos $(5,14,20)$. En este estudio tomamos como punto de corte un valor menor de 26 , basados en el trabajo de Blumel et al. en Chile, encontrando una prevalencia de disfunción sexual del 60,2\% (12).

Con respecto a la escolaridad y su papel en la aparición de disfunción sexual durante el embarazo, no encontramos diferencias. Algunos reportes determinaron que la diferencia en la proporción de disfunción sexual es más significativa entre pacientes analfabetas y las que terminaron su bachillerato (17). Sin embargo, en una revisión realizada en 1999, donde se evaluaron 59 estudios, los resultados con respecto a la educación de las pacientes y la presencia de disfunción sexual no fueron concluyentes (10).

El factor que presentó mayor afectación fue el alcance del orgasmo, casi la mitad de nuestras pacientes tuvieron dificultad para lograrlo, a pesar de que el $49 \%$ se sintieron satisfechas con sus orgasmos, con una lubricación adecuada en la misma proporción, incluso al final de 
la relación. En general nuestras pacientes reportaron satisfacción en la cercanía de su pareja, aunque solo un tercio se sentían satisfechas con su vida sexual (Figura 2). Según lo reportado en otras revisiones, esta satisfacción puede variar según el trimestre, encontrando 59\% durante el primer trimestre, $75 \%$ durante el segundo y $40 \%$ durante el tercer trimestre (18). La sensación de dolor durante el coito es también un factor que puede aportar a la disfunción sexual, el cual ha sido reportado con una frecuencia entre el 30 al $50 \%$ en otras revisiones $(18,21,22)$, resultado consistente con los hallazgos del estudio donde se presentó en el $37 \%$ de las pacientes.

Es importante tener en cuenta que la presencia de disfunción sexual no es solo una patología que se presente durante el embarazo; por ejemplo, un estudio realizado en Turquía, donde se evaluaron 282 mujeres jóvenes, sanas no gestantes; se reportó que el 53,2\% presentaron un IFSF menor a 26, clasificándolas como disfunción sexual (23). Con esto se evidencia que es un problema médico altamente prevalente, que afecta de forma significativa la vida sexual y reproductiva de las mujeres, con múltiples posibles factores de riesgo $(24,25)$, y que los profesionales de la salud deben estar alerta para detectarlos de forma temprana y así evitar que se desarrolle esta condición.

Entre las limitaciones del estudio está la no discriminación por trimestre, lo cual se ha reportado en otras revisiones, como la realizada en 2007 por Erol B et al., donde se incluyeron 589 pacientes gestantes sanas, donde se evaluó el IFSF, con un punto de corte de 30 , evidenciando scores menores en las mujeres en el tercer trimestre, hallazgo que se repite en la mayoría de los estudios $(14,17)$ o comparar los resultados con población femenina no gestante para atribuir este fenómeno al embarazo. Se realizó un muestreo por conveniencia y no se incluyeron pacientes en nivel socioeconómico alto, por lo que no se puede extrapolar la información obtenida a este grupo poblacional, con un potencial un sesgo de selección. Además, algunas encuestas fueron respondidas de manera incompleta probablemente por los mitos o miedos de las pacientes para hablar sobre el tema, produciendo un sesgo de información. Las asociaciones encontradas fueron crudas y no se consideró el efecto de factores de confusión de dichas asociaciones; considerando este aspecto, su interpretación debe sercautelosa.

Este es el primer estudio sobre este tema en nuestro medio, además de su carácter multicéntrico, dando una primera impresión de la disfunción sexual en las pacientes embarazadas, siendo posiblemente el primer paso para desarrollo de estudios posteriores.

\section{CONCLUSIÓN}

La disfunción sexual es una entidad con una prevalencia importante en las mujeres, y el embarazo es una etapa en la cual puede ser más frecuente. Es importante que los profesionales de la salud estén sensibilizados con esta alteración y brinden de manera adecuada información clara a las pacientes sobre la seguridad de las relaciones sexuales durante un embarazo de bajo riesgo y los posibles cambios en el deseo, el alcance del orgasmo y la satisfacción sexual que se pueden presentar.

\section{CONFLICTOS DE INTÉRES}

Los autores declaran su independencia con las instituciones financiadoras y de apoyo y que durante la redacción del manuscrito no incidieron intereses o valores distintos a los de la investigación.

\section{REFERENCIAS}

1. Read J. ABC of sexual health: Sexual problems associated with infertility, pregnancy, and ageing. BMJ. 2004; 329(7465):559-61. https://doi.org/10.1136/ bmj.329.7465.559

2. Pauleta JR, Pereira NM, Graca LM. Sexuality During Pregnancy. J Sex Med. 2010; 7(1):136-42. https://doi. org/10.1111/j.1743-6109.2009.01538.x

3. Serati M, Salvatore S, Siesto G, Cattoni E, Zarinato M, KhullarV, etal.Female sexualfunction during pregnancy and after childbirth. J Sex Med. 2010; 7(8): 2782-90. https://doi.org/10.1111/j.1743-6109.2010.01893.x

4. Johnson CE. Sexual Health during Pregnancy and the Postpartum. J Sex Med. 2011; 8(5): 1267-84. https:// doi.org/10.1111/j.1743-6109.2011.02223.x

5. Connolly A, Thorp J, Pahel L. Effects of pregnancy and childbirth on postpartum sexual function: A longitudinal prospective study. Int Urogynecol J. 2005; 16: 263-7. https://doi.org/10.1007/s00192-005-1293-6

6. Barrett G, Pendry E, Peacock J, Victor C, Thakar R, Manyonda I. Women's sexual health after childbirth. BJOG: An International Journal of Obstetrics \& Gynaecology. 2000; 107(2): 186-95. https://doi. org/10.1111/j.1471-0528.2000.tb11689.x

7. Basson R. Women's sexual dysfunction: Revised and expanded definitions. CMAJ 2005; 172(10): 1327-33. https://doi.org/10.1503/cmaj.1020174 
8. Erol B, Sanli 0, Korkmaz D, Seyhan A, Akman T, Kadioglu A. A Cross-Sectional Study of Female sexual Function and Dysfunction During Pregnancy.J Sex Med. 2007; 4(5):1381-7. https://doi.org/10.1111/j.17436109.2007.00559.x

9. Bartellas E, Crane JM, Daley M, Bennett KA, Hutchens D. Sexuality and sexual activity in pregnancy. BJOG: An InternationalJournalofObstetrics\&Gynaecology.2005; 107: 964-8. https://doi.org/10.1111/j.1471-0528.2000. tb10397.x

10. Kucukdurmaz F, Efe E, Malkoc O, Kolus E, Amasyali AS, Resim S. Prevalence and correlates of female sexual dysfunction among Turkish pregnant women. Turk J Urol. 2016; 42(3):178-83. doi: https://doi.org/10.5152/ tud.2016.49207

11. von Sydow K. Sexuality during pregnancy and after childbirth: A metacontent analysis of 59 studies. J Psychosom Res. 1999; 47(1): 27-49. https://doi. org/10.1016/S0022-3999(98)00106-8

12. Blümel JE, Binfa L, Cataldo P, Carrasco A, Izaguirre H, Sarrá $S$. Índice de función sexual femenina: una prueba para evaluar la sexualidad de la mujer. Rev Chil Obstet Ginecol. 2004; 69(2): 118-25. https://doi.org/10.4067/ S0717-75262004000200006.

13. Stephenson KR, Toorabally N, Lyons L, M. Meston C. Further Validation of the Female Sexual Function Index: Specificity and Associations with Clinical Interview Data.Journal of Sex \& Marital Therapy. 2016; 42(5):44861. https://doi.org/10.1080/0092623X.2015.1061078

14. Polomeno V. Sex and pregnancy: A perinatal educator's guide. J Perinat Educ. 2000; 9(4): 15-27. doi: https:// doi.org/10.1624/105812400X87879

15. Hart J, Cohen E, Gingold A, Homburg R. Sexual behavior in pregnancy: A study of 219 women. Journal of Sex Education and Therapy. 2015; 17(2): 86-90. https://doi. org/10.1080/01614576.1991.11074009

16. Rosen CB. The Female Sexual Function Index (FSFI): a multidimensional self-report instrument for the assessment of female sexual function. Journal of Sex \& Marital Therapy. 2000; 26(2): 191-208. https://doi. org/10.1080/009262300278597.

17. Sacomori C, Cardoso FL. Sexual Initiative and Intercourse Behavior During Pregnancy Among Brazilian Women: A Retrospective Study. Journal of Sex \& Marital Therapy. 2010; 36(2): 124-36. https://doi. org/10.1080/00926230903554503.

18. Byrd JE, Hyde JS, DeLamater JD, Plant EA. Sexuality during pregnancy and the year postpartum. J Fam Pract. 1998; 47: 305-8.

19. Paterson L, Davis SN, Khalife S, Amsel R, Binik YM. Persistent genital pain and pelvic pain after childbirth.
J Sex Med. 2009; 6(1): 215-21. https://doi.org/10.1111/ j.1743-6109.2008.01063.x

20. Signorello LB, Harlow BL, Chekos AK, Repke JT. Postpartum sexual functioning and its relationship to perineal trauma: A retrospective cohort study of primiparous women. AmJ Obstet Gynecol. 2001; 184(5): 881-90. https://doi.org/10.1067/mob.2001.113855

21. Serati M, Salvatore S, Khullar V, Uccella S, Bertelli E, Ghezzi F, Bolis P. Prospective study to assess risk factors for pelvic floor dysfunction after delivery. Acta Obstetricia et Gynecologica Scandinavica. 2008; 87:3138. doi: https://doi.org/10.1080/00016340801899008

22. Avery MD, Duckett L, Frantzich CR. The Experience of Sexuality During Breastfeeding Among Primiparous Women. Journal of Midwifery \& Women's Health. 2000; 45(3): 227-37. https://doi.org/10.1016/S15269523(00)00020-9

23. Kllç M. Prevalence and risk factors of sexual dysfunction in healthy women in Turkey. Afr Health Sci. 2019; 19(3): 2623-33. doi: https://doi.org/10.4314/ahs.v19i3.38

24. McCool-Myers M, Theurich M, Zuelke A, Knuettel H, Apfelbacher C. Predictors of female sexual dysfunction: a systematic review and qualitative analysis through gender inequality paradigms. BMC Women's Health. 2018; 108. https://doi.org/10.1186/s12905-018-0602-4

25. Moreau C, KÍgesten AE, Blum RW. Sexual dysfunction among youth: an overlooked sexual health concern. BMC Public Health. 2016; 16:1170. https:/doi. org/10.1186/s12889-016-3835-x 